\title{
COMPARISON OF SHAPE DERIVATIVES USING CUTFEM FOR ILL-POSED BERNOULLI FREE BOUNDARY PROBLEM
}

\author{
Cuiyu He*, Erik Burman ${ }^{\dagger}$ and Mats G. Larson ${ }^{\dagger}$ \\ * University of Georgia \\ Athens, GA, USA, 30677 \\ e-mail: cuiyu.he@uga.edu,web page: https://sites.google.com/view/cuiyu-he/home \\ $\dagger$ University College London \\ Department of Mathematics, Gower Street, London, WC1E 6BT \\ e-mail: e.burman@ucl.ac.uk \\ Umea University \\ MIT-huset, plan 3, Matematik och matematisk statistik, D354 Umeäuniversitet, 90187 Umeä \\ email: mats.larson@umu.se
}

\begin{abstract}
In this paper we study and compare three types of shape derivatives for free boundary identification problems. The problem takes the form of a severely ill-posed Bernoulli problem where only the Dirichlet condition is given on the free (unknown) boundary, whereas both Dirichlet and Neumann conditions are available on the fixed (known) boundary. Our framework resembles the classical shape optimization method in which a shape dependent cost functional is minimized among the set of admissible domains. The position of domain is defined implicitly by the level set function. The gradient (shape derivative) descent method is applied for the level set evolution. Numerically, we choose the Cut finite element method (CutFEM), that circumvents meshing and re-meshing, to obtain high accuracy approximations of the involving partial differential models.
\end{abstract}

